\title{
Comparative analysis of serum proteomes: Identification of proteins associated with sciatica due to lumbar intervertebral disc herniation
}

\author{
PEIGEN XIE, BIN LIU, RUIQIANG CHEN, BU YANG, JIANWEN DONG and LIMIN RONG \\ Department of Spine Surgery, The Third Affiliated Hospital of Sun Yat-sen University, \\ Guangzhou, Guangdong 510630, P.R. China
}

Received February 24, 2014; Accepted May 19, 2014

DOI: 10.3892/br.2014.295

\begin{abstract}
Lumbar intervertebral disc herniation (LDH) is one of the most common orthopedic conditions that can cause lower back pain and sciatica. However, the pathogenesis of LDH is poorly understood. The aim of the present study was to use proteomic analysis of blood samples to establish whether there are serum proteins associated with LDH, which may be useful in elucidating LDH pathogenesis. The ultimate aim was to develop a simple technique for the diagnosis of $\mathrm{LDH}$ based on the blood samples of patients with sciatica. The study used comparative analysis of serum proteomes associated with sciatica due to LDH. A total of $30 \mathrm{LDH}$ patients with sciatica, receiving treatment between August and December 2007, were selected as the experimental group (or LDH group). A total of $2 \mathrm{ml}$ of blood was obtained from each of the 30 patients in the LDH group and from 30 healthy volunteers, who constituted the control group. Two-dimensional electrophoresis of the blood samples was conducted, distinct protein spots were identified by matrix-assisted laser desorption/ionization time-of-flight mass spectrometry and proteins associated with LDH were detected. An enzyme-linked immunosorbent assay (ELISA) was developed to screen for the LDH proteins and was tested on the sera of a second test and control group that included 10 patients with LDH and 10 healthy subjects, respectively. Based on signal intensity, the expression levels of 6 proteins on the dielectrophoretogram were found to be significantly associated with LDH. The identities of the LDH proteins were upregulated apolipoprotein-L1 (APO-L1) and two types of serum albumin precursors, and downregulated
\end{abstract}

Correspondence to: Professor Limin Rong, Department of Spine Surgery, The Third Affiliated Hospital of Sun Yat-sen University, 600 Tianhe Road, Tian He, Guangzhou, Guangdong 510630, P.R. China

E-mail: ronglimin@21cn.com

Key words: lumbar intervertebral disc herniation, sciatica, proteomics, apolipoprotein-L1, tetranectin, apolipoprotein M, immunoglobulin light chain apolipoprotein M (APO-M), tetranectin (TN) and immunoglobulin light chain (IGL). Further ELISA experiments confirmed that there were increased serum levels of 4 out of the 6 proteins in patients with sciatica due to $\mathrm{LDH}$, which was statistically different compared to the healthy subjects. In conclusion, these results suggest that serum APO-L1, TN, APO-M and IGL may serve as LDH biomarkers.

\section{Introduction}

Lumbar intervertebral disc herniation (LDH) is one of the most common orthopedic conditions that can cause lower back pain and sciatica. It is estimated that $\sim 70 \%$ of the population experiences lower back pain during their lifespan, and at any given time, 55\% suffer from lower back pain associated with radicular syndromes (1-3). Sciatica symptoms are extremely persistent and up to one-third of all patients with sciatica undergo lumbar surgery (4). A number of studies have discussed the mechanical and biochemical factors involved in the induction of radicular pain due to LDH (5) and numerous trials of potential molecular and biological therapies have been conducted in this context. Previous studies have evaluated three main biochemical changes: Decreased matrix synthesis, increased catabolism, and changed levels of growth factors and cytokines associated with altered disc cell phenotypes during disc aging and degeneration (6-11). However, sciatica of a radicular origin represents an undetermined clinical problem. The study by Bogduk (12) stated that discogenic pain cannot be diagnosed clinically and relies on supposition. Thus far, the pathogenesis of LDH is poorly understood and, aside from surgical intervention, no effective therapy is available.

Proteomic techniques are powerful tools that can provide detailed information regarding changes to the expression of protein profiles. Studies on proteomics have been carried out in certain diseases, including malignant tumors and neuropathy (13-17). A specific marker for a particular disease can be identified by comparing the protein spectrum of a patient with the disease with that of a healthy subject or with the protein spectrum from the gene bank (18). Proteomics may become one of the best measures for tracking disease markers and drug targets. Although there are certain studies describing the use of proteomics for marker detection and evaluation of 
pathogenesis in rheumatoid arthritis, few studies have been published regarding proteomics research in the osteoarthritis system $(19,20)$, particularly in LDH. The pathogenesis and development of LDH undergoes a multi-stage process, which is based on gene expression changes. Proteins are the key bridges that connect genes with the biological function outcomes. Therefore, more proteomic studies are required to help improve the understanding of LDH pathogenesis. Proteomic analysis studies of the cerebrospinal fluid (CSF) of patients with LDH have documented quantitative differences in the expression of CSF proteins from patients with LDH compared to controls. Thus, these changes can be considered to be biomarkers of a condition, such as LDH (21). However, it is difficult to obtain the CSF and therefore it is difficult to identify such biomarkers. Therefore, the aim of the present study was to use proteomics to identify differentially expressed proteins in the sera of LDH patients compared to control subjects. The validity of the differential protein expression in the patients with LDH was further examined and confirmed with an enzyme-linked immunosorbent assay (ELISA). We suggest that these differentially expressed proteins may be useful as biomarkers for $\mathrm{LDH}$, and in addition, they may lead to the development of targeted therapies for this disease.

\section{Materials and methods}

Diagnostic criteria for $\mathrm{LDH}$. The diagnosis of $\mathrm{LDH}$ was based on the symptoms and objective signs of sciatica. Lower back pain, severe lower leg pain and the Lasègue sign were observed in all the patients. In addition, magnetic resonance imaging confirmed LDH. Subjects with liver or kidney diseases were excluded from the study. Three orthopedic surgeons conducted independent clinical diagnoses on the basis of the physical and radiological examinations, which were confirmed by surgery.

Subjects. A total of 30 patients with LDH (15 females and 15 males, 28.1 \pm 2.3 years old, who had never received any prior surgical treatment and had not possessed any disease-related or predisposing risk for LDH) and 30 healthy subjects who lacked any symptoms of disc herniation (15 females and 15 males, 25.2 \pm 3.1 years old) were included in the proteomics study. Another 10 patients with LDH (5 females and 5 males, $26.3 \pm 2.3$ years old) and 10 healthy subjects ( 5 females and 5 males, 30.1 \pm 3.3 years old) were enrolled for the ELISA test.

Serum samples. Venous blood samples were collected from all subjects and phenylmethylsulfonyl fluoride was added to the samples. The serum was separated from the samples and stored at $-80^{\circ} \mathrm{C}$ until use according to the instructions of the ProteoPrep Blue Albumin and IgG Depletion kit (Sigma) and 2-D clean up kit (Amersham Biosciences). To minimize the variation among individuals, the gender and age of the patients were matched in the control and LDH groups in the proteomic study. The study was approved by the local ethics committee of the Third Affiliated Hospital of Sun Yat-sen University and it was carried out in compliance with the principles stipulated by the Declaration of Helsinki. All patients and control subjects gave their informed consent prior to enrollment in the study.

A 30- $\mu 1$ serum sample was loaded onto a 1-ml column. According to the manufacturer's instructions, a
ProteoPrep $^{\circledR}$ Blue Albumin and immunoglobulin G (IgG) Depletion kit (Sigma-Aldrich, St. Louis, MO, USA) was used to remove serum albumin and IgG. A 2-D clean-up kit (Amersham Biosciences Corp., Piscataway, NJ, USA) was utilized to discard the salt and sulfatide in the serum. Protein concentrations in the serum were determined prior to conducting two-dimensional electrophoresis (2-DE), using Bradford's method (22) with bovine serum albumin as the standard.

Two-dimensional polyacrylamide gel electrophoresis (2D-PAGE). 2D-PAGE was performed according to the method of Görg et al (23) and the manufacturer's instructions of the Bio-Rad (Hercules, CA, USA) electrophoresis device. Non-linear immobiline $\mathrm{pH}$ 4-7 gradient (IPG) strips (Bio-Rad) were used for isoelectric focusing (IEF). A 0.3-mg sample of the total protein was loaded onto the IPG strips. The strips were rehydrated at $50 \mathrm{~V}$ for $12 \mathrm{~h}$, followed by IEF at $150 \mathrm{~V}$ for $1 \mathrm{~h}, 500 \mathrm{~V}$ for $3 \mathrm{~h}, 1,000 \mathrm{~V}$ for $1 \mathrm{~h}, 5,000 \mathrm{~V}$ for $1 \mathrm{~h}, 7,000 \mathrm{~V}$ for $2 \mathrm{~h}$ and $10,000 \mathrm{~V}$ until the total voltage $\mathrm{x}$ hours of exposure reached 50,000 Vh. Following this, the strips were equilibrated for $15 \mathrm{~min}$ in equilibration buffer I [6 $\mathrm{M}$ urea, $2 \%$ sodium dodecyl sulfate, $50 \mathrm{M}$ Tris- $\mathrm{HCl}$ ( $\mathrm{pH} 8.8$ ), 30\% glycerol, $2 \%$ dithiothreitol (DTT) and bromophenol blue]. The strips were subsequently equilibrated in the same buffer containing $3 \%$ iodoacetamide instead of DTT for $15 \mathrm{~min}$, and were then transferred to a $12 \%$ glycerol gradient gel for separation. The second dimensional separation was performed at $12 \mathrm{~mA}$ per strip for $30 \mathrm{~min}$, subsequently switching to $20 \mathrm{~mA}$ per strip until the bromophenol blue indicator reached the bottom of the gel. Following 2-DE, the proteins in the gel were visualized by silver staining (20).

Image analysis. The stained gel was scanned by an Image Scanner type II (Amersham Biosciences Corp.) and the 2-D electrophoregram was analyzed with the application of ImageMaster 2D Elite 5.0 software (Amersham Biosciences Corp.). The data were statistically processed on the Windows program SPSS v15.0 (SPSS, Inc., Chicago, IL, USA). P<0.05 was considered to indicate a statistically significant difference.

Identification of proteins by matrix-assisted laser desorption/ionization (MALDI) time-of-flight (TOF) mass spectrometry $(M S)$. Protein spots exhibiting significant expressional differences (up- or downregulated by $>3$-fold) were excised from the gel and sliced into sections of $\sim 1 \mathrm{~mm}^{3}$. The spots were destained and dehydrated. Trypsin digestion solution, which was treated with $\mathrm{N}$-tosyl-L-phenylalanine chloromethyl ketone, was added to the tube containing the spots for digestion overnight at $37^{\circ} \mathrm{C}$. Following digestion, the peptides were obtained from the extraction buffer, which contained $50 \%$ acetonitrile and $0.1 \%$ trifluoroacetic acid (TFA), and were dried under $\mathrm{N}_{2}$. The extracted peptides were mixed with $\alpha$-cyano-4-hydroxycinnamic acid solution in $0.1 \%$ TFA and $50 \%$ acetonitrile. The solution obtained was placed on the metal plate of the MALDI-TOF target and dried at room temperature. The samples were analyzed using MALDI-TOF-MS (Applied Biosystems, Foster City, CA, USA). The spectrum was recorded in the reflector positive ion mode. The standard conditions consisted of a 355-nm 
Table I. Matrix-assisted laser desorption/ionization time-of-flight mass spectrometry analysis of differentially expressed proteins in patients with lumbar intervertebral disc herniation $(\mathrm{LDH})$ compared to healthy controls.

\begin{tabular}{lclcccc}
\hline Spot no. & Accession no. & \multicolumn{1}{c}{ Protein name } & MW, Da & pI & $\begin{array}{c}\text { Protein } \\
\text { score }\end{array}$ & $\begin{array}{c}\text { Optical density } \\
\text { intensity in LDH }\end{array}$ \\
\hline A1 & 645213 & Apolipoprotein M & 13042.4 & 7.66 & 210 & Decreased \\
A2 & 796167 & Immunoglobulin light chain & 14669.1 & 6.25 & 207 & Decreased \\
A3 & 384697 & Serum albumin precursor & 47329.7 & 5.97 & 371 & Increased \\
A4 & 745872 & Serum albumin precursor & 69321.5 & 5.92 & 107 & Increased \\
A5 & 9028 & Tetranectin & 22552.3 & 5.52 & 307 & Decreased \\
A6 & 514475 & Apolipoprotein L1 & 43900.0 & 5.84 & 234 & Increased \\
\hline
\end{tabular}

MW, molecular weight; and pI, isoelectric point.

wavelength laser and an accelerating voltage of $20,000 \mathrm{~V}$. The matrix and peptides were selected in the mass range of 700-3,500 Da. Subsequent to MS analysis, the peptides that were different from the matrix peptide mass fingerprint were selected and the mass spectrum/mass spectrum was achieved.

Database retrieval. The mass spectrum obtained from the MALDI-TOF was assessed by searching the Mascot software from NCBInr (www.matrixscience.com) with the following restrictions to the initial retrieval: Species, Homo Sapiens; mass range, 800-4,000 Da; isoelectric point (pI) range, 4-7; maximum tolerance error of peptide fragment, $\pm 0.5 \mathrm{Da}$; ion selection, $(\mathrm{M}+\mathrm{H})^{+}$and monoisotopic; a minimum of 4 matched peptides; fixed modifications, carbamidomethyl; and variable modifications, oxidation.

ELISA. The levels of the aforementioned identified proteins (associated with LDH pathology) were measured in the blood serum samples using an ELISA kit (BMA Biomedicals AG, Augst, Switzerland) according to the manufacturer's instructions. The blood serum was obtained from another 10 patients with LDH and 10 healthy subjects.

\section{Results}

Analysis of the protein profiles. The protein profiles from the sera of 30 patients with $\mathrm{LDH}$ and 30 healthy subjects were analyzed by 2-DE with silver-staining of proteins in the gel. Several protein spots were separated on 2-DE gels from

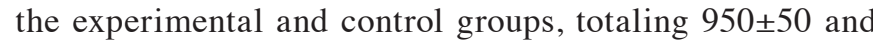
$920 \pm 50$ spots, respectively. The majority of protein spots were clustered between 10 and $120 \mathrm{kDa}$ and between $\mathrm{pI} 4-7$. The overall pattern of the protein expression in all 2-DE gels was extremely similar. The proteins that were up- or downregulated by $>3$-fold and appeared in all patients with LDH pathology were selected for identification by peptide mass fingerprinting, using MALDI-TOF-MS and protein database searching. The results from this identification were of high confidence if the protein had a significant score and high-sequence coverage. A total of 6 protein spots were identified as being associated with LDH pathology (Table I). The identities of these 6 spots were found to be upregulated apolipoprotein-L1 (APO-L1) and two serum albumin precursors, and downregulated apolipoprotein M (APO-M), tetranectin (TN) and immunoglobulin light chain (IGL). The 6 proteins were found to be significantly and consistently different in the sera of patients with LDH compared to healthy subjects, according to 2-DE gel separation and proteomic analysis (Fig. 1).

The protein expression of APO-L1 was not detected in the sera of healthy subjects but was only found in patients with LDH pathology. In addition, the protein expression levels of APO-M, TN and IGL in the sera from patients with LDH were all downregulated by $22 \pm 3(\mathrm{P}<0.01), 37 \pm 5(\mathrm{P}<0.01)$ and $27 \pm 3 \%(\mathrm{P}<0.01)$, respectively, compared to the sera from healthy subjects.

The ELISA results support the proteomic analysis results. The ELISA demonstrated that the mean serum concentrations of APO-M, TN and IGL were significantly lower in LDH patients compared to the healthy control group $(\mathrm{P}<0.05)$. By contrast, the plasma levels of APO-L1 in LDH patients were significantly higher than the values recorded for the healthy control group $(\mathrm{P}<0.01)$.

\section{Discussion}

Proteomics is a powerful tool in the analysis of protein expression and composition of various tissues, as it provides useful information regarding biochemical pathways and biological processes and connects the relevant genes with protein expression. Proteomics primarily involves the application of 2-DE, MS and database retrieval systems (24). Due to its reproducibility, accuracy and high-throughput, proteomics has been extensively used in studies pertaining to tumors, inflammation and immunity, as well as other complex areas of study. Although proteomics is becoming increasingly available to investigators, the application of proteomics to $\mathrm{LDH}$ is only in the initial stages (21).

In the present study, proteomics was applied to identify specific biomarkers associated with the pathogenesis of $\mathrm{LDH}$. A total of 6 proteins were found that were differentially expressed in the sera of 30 patients with LDH compared to 30 healthy subjects. Furthermore, 4 of these 6 proteins were statistically different in terms of their serum level between the experimental and control groups as determined by the ELISA measurements on the serum samples. These 4 proteins were APO-L1, TN, APO-M and IGL. 

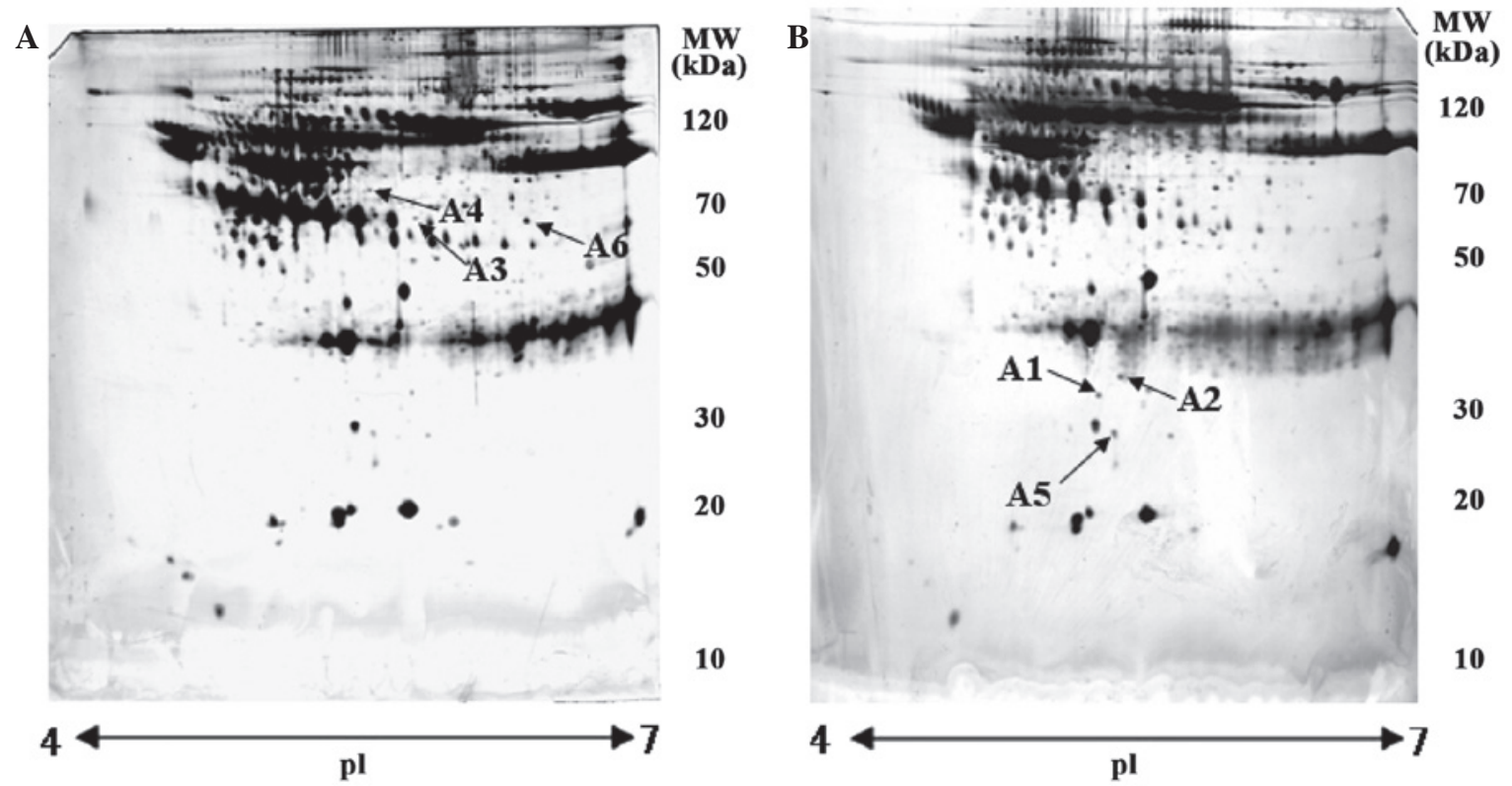

Figure 1. Human serum proteins separated on two-dimensional electrophoresis gels; (A) from a patient with LDH and (B) a healthy control. Total protein $(300 \mu \mathrm{g}$ ) was loaded on the immobiline $\mathrm{pH}$ gradient strip ( $\mathrm{pH} 4-7$, non-linear) for isoelectric focusing and separated in the second dimension. Subsequently, the spots were silver-stained. The arrows indicate 6 distinctly expressed protein spots. LDH, lumbar intervertebral disc herniation.

Extremely little is known with regard to APO-L, a protein first characterized in 1997 as a new human high-density lipoprotein (25). APO-L was found only in the pancreas and is described as a 383 -amino acid residue protein with no significant homology to any other known protein sequence. In healthy subjects, free APO-L is not detected in the plasma; instead, it binds to other proteins, mainly large APO-A1-containing lipoproteins. The cloning of CG12_1 in 1999 suggested that other APO-L proteins may exist. APO-L is specifically expressed in endothelial cells lining healthy atherosclerotic iliac arteries and the aorta, and it is responsive to changes in the tumor necrosis factor (TNF)- $\alpha$ level (26). The study by Horrevoets et al (26) also identified and classified novel members of the APO-L protein family. The similarities of these proteins suggest that they have arisen through local gene duplication. Although they do not possess classical signal peptide sequences, evidence exists that at least APO-L1 is secreted in the plasma $(25,27)$. It is believed that TNF- $\alpha$ plays a key role in the inflammation induced by LDH (28-30). However, Brisby et al (31) found that the concentration of TNF- $\alpha$ in the serum of LDH patients is not significantly different compared to healthy patients. Therefore, the level of APO-L1 may be a reflection of the TNF- $\alpha$ secreted into the serum and may serve as a biomarker for LDH. Additionally, APO-L1 is associated with the lysis of lysosomes and the appearance of an APO-L version with a signal peptide represents a novel component of innate immunity $(32,33)$. Herniation of the intervertebral disc is associated with human autoimmunity. In addition, APO-L1 is a novel Bcl-2 homology domain 3-only protein, which when overexpressed and accumulated intracellularly, induces autophagic cell death in cells as characterized by the increasing formation of autophagic vacuoles (34). Thus, APO-L1 may be the signal peptide of LDH.

$\mathrm{TN}$ is a plasminogen-binding protein, that can be encountered in the plasma and in the extracellular matrix
(ECM) $(35,36)$. In addition to its plasminogen-binding properties, TN can bind to heparin, APO-A, tissue plasminogen activator, hepatocyte growth factor and angiostatin (37-39). $\mathrm{TN}$ expression has been detected in various endocrine tissues, as well as in epithelial and mesenchymal cells, including fibroblasts, monocytes and neutrophils (40-42). TN also constitutes a crucial component of the ECM in osteogenesis, muscle development and regeneration, suggesting its important physiological role in tissue remodeling $(43,44)$. Serum TN is decreased following trauma or acute myocardial infarction, during pregnancy and in patients with liver cirrhosis, rheumatoid arthritis or malignant tumors $(37,45-47)$. Although the exact biological function of $\mathrm{TN}$ has yet to be established, certain evidence suggests that TN plays an important role in tissue remodeling. TN increases the tissue-type plasminogen activator-catalyzed activation of plasminogen in the presence of poly-d-lysine (35) and activated plasminogen is considered to play a key role in the degradation of the ECM. In this study, serum TN was also decreased. As the majority of the LDH cases are caused by degeneration of the lumbar disc, in the process of which apoptosis of the nucleus pulposus cell is first observed and is subsequently followed by the imbalance of lumbar disc remodeling. In this way, the serum TN level is reduced in patients with $\mathrm{LDH}$, which is considered to be associated with regeneration of the lumbar disc, leading to a decrease in the synthesis and secretion of TN.

Human APO-M was found and initially isolated from chylomicrons in the 1999 study by Xu and Dahlbäck (48). $\mathrm{Xu}$ et al (49) have reported that transforming growth factor $\beta$ can also downregulate APO-M expression and secretion in HepG2 cells. These data indicate that APO-M may be associated with the host defense response as the APO-M gene is located in the histocompatibility complex III region on chromosome 6 (50). A number of genes in this region are associated with the immune responses and the APO-M 
gene is extremely close to the TNF- $\alpha$ gene and lymphotoxin genes (50). Therefore, APO-M may also be associated with the immune system, or regulated by cytokines or other inflammatory factors. The detailed association between APO-M and $\mathrm{LDH}$ remains unclear and requires further investigation. The function of IGL in LDH patients also remains unclear. Thus, it can be used clinically for screening or monitoring $\mathrm{LDH}$ requires further studies.

Previously, no direct association between the pathogenesis of $\mathrm{LDH}$ and the 4 proteins that were differentially expressed in the LDH group has been found. To the best of our knowledge, the present study is the first to report on this association and is unique as the serum molecular analysis of LDH was accomplished using a proteomics approach. Thus, proteomic analysis is an efficient technique for identifying the diagnostic biomarkers of LDH. In conclusion, the 4 proteins that were differentially expressed in LDH patients compared to healthy subjects were recognized. These findings contribute to the advancement of knowledge in this area of study.

\section{References}

1. Macfarlane GJ, Thomas E, Croft PR, et al: Predictors of early improvement in low back pain amongst consulters to general practice: the influence of pre-morbid and episode-related factors. Pain 80: 113-119, 1999.

2. Frymoyer JW: Lumbar disc disease: epidemiology. Instr Course Lect 41: 217-223, 1992.

3. Long DM: Decision making in lumbar disc disease. Clin Neurosurg 39: 36-51, 1992.

4. Balagué F, Nordin M, Shelkhzadeh A, et al: Recovery of severe sciatica. Spine (Phila Pa 1976) 24: 2516-2524, 1999.

5. Igarashi T, Kikuchi S, Shubayev V, et al: 2000 Volvo Award winner in basic science studies: Exogenous tumor necrosis factor-alpha mimics nucleus pulposus-induced neuropathology. Molecular, histologic, and behavioral comparisons in rats. Spine (Phila Pa 1976) 25: 2975-2980, 2000.

6. Yoon ST and Patel NM: Molecular therapy of the intervertebral disc. Eur Spine J 15 (Suppl 3): S379-S388, 2006.

7. Kuh SU, Zhu Y, Li J, et al: A comparison of three cell types as potential candidates for intervertebral disc therapy: annulus fibrosus cells, chondrocytes, and bone marrow derived cells. Joint Bone Spine 76: 70-74, 2009.

8. Zhao CQ, Wang LM, Jiang LS and Dai LY: The cell biology of intervertebral disc aging and degeneration. Ageing Res Rev 6: 247-261, 2007.

9. Diefenderfer DL and Brighton CT: Microvascular pericytes express aggrecan message which is regulated by BMP-2 Biochem Biophys Res Commun 269: 172-178, 2000.

10. Tim Yoon S, Su Kim K, Li J, et al: The effect of bone morphogenetic protein-2 on rat intervertebral disc cells in vitro. Spine (Phila Pa 1976) 28: 1773-1780, 2003.

11. Fassett DR, Kurd MF and Vaccaro AR: Biologic solutions for degenerative disc disease. J Spinal Disord Tech 22: 297-308, 2009

12. Bogduk N: What's in a name? The labelling of back pain. Med J Aust 173: 400-401, 2000 .

13. Wattiez R, Hermans C, Bernard A, Lesur O and Falmagne P: Human bronchoalveolar lavage fluid: two-dimensional gel electrophoresis, amino acid microsequencing and identification of major proteins. Electrophoresis 20: 1634-1645, 1999.

14. Saintigny G, Schmidt R, Shroot B, Juhlin L, Reichert U and Michel S: Differential expression of calgranulin A and B in various epithelia cell lines and reconstructed epidermis. J Invest Dermatol 99: 639-644, 1992.

15. Ruebelt MC, Lipp M, Reynolds TL, et al: Application of two-dimensional gel electrophoresis to interrogate alterations in the proteome of genetically modified crops. 3. Assessing unintended effects. J Agric Food Chem 54: 2169-2177, 2006.

16. Lee EG, Kim JH, Shin YS, et al: Application of proteomics for comparison of proteome of Neospora caninum and Toxoplasma gondii tachyzoites. J Chromatogr B Analyt Technol Biomed Life Sci 815: 305-314, 2005.
17. Tan Y, Lv ZP and Zhang XF: Proteome and its application in liver disease research. Zhonghua Gan Zang Bing Za Zhi 12: 700-702, 2004 (In Chinese).

18. Nomura F, Tomonaga T, Sogawa K, et al: Identification of novel and downregulated biomarkers for alcoholism by surface enhanced laser desorption/ionization-mass spectrometry. Proteomics 4: 1187-1194, 2004.

19. Tilleman K, Van Beneden K, Dhondt A, et al: Chronically inflamed synovium from spondyloarthropathy and rheumatoid arthritis investigated by protein expression profiling followed by tandem mass spectrometry. Proteomics 5: 2247-2257, 2005.

20. Tan X, Cai D, Wu Y, et al: Comparative analysis of serum proteomes: discovery of proteins associated with osteonecrosis of the femoral head. Transl Res 148: 114-119, 2006.

21. Liu XD, Zeng BF, Xu JG, et al: Proteomic analysis of the cerebrospinal fluid of patients with lumbar disc herniation. Proteomics 6: 1019-1028, 2006.

22. Bradford MM: A rapid and sensitive method for the quantitation of microgram quantities of protein utilizing the principle of protein-dye binding. Anal Biochem 72: 248-254, 1976.

23. Görg A, Obermaier C, Boguth G, et al: The current state of two-dimensional electrophoresis with immobilized $\mathrm{pH}$ gradients. Electrophoresis 21: 1037-1053, 2000.

24. Wilkins MR, Williams KL, Appel RD, Hochstrasser DF (eds): Proteome Research: New Frontiers in Functional Genomics. Springer, Heidelberg, pp188-219, 1997.

25. Duchateau PN, Pullinger CR, Orellana RE, et al: Apolipoprotein L, a new human high density lipoprotein apolipoprotein expressed by the pancreas. Identification, cloning, characterization, and plasma distribution of apolipoprotein L. J Biol Chem 272: 25576-25582, 1997.

26. Horrevoets AJ, Fontijn RD, van Zonneveld AJ, et al: Vascular endothelial genes that are responsive to tumor necrosis factor-alpha in vitro are expressed in atherosclerotic lesions, including inhibitor of apoptosis protein-1, stannin, and two novel genes. Blood 93: 3418-3431, 1999.

27. Duchateau PN, Movsesyan I, Yamashita S, et al: Plasma apolipoprotein $\mathrm{L}$ concentrations correlate with plasma triglycerides and cholesterol levels in normolipidemic, hyperlipidemic, and diabetic subjects. J Lipid Res 41: 1231-1236, 2000.

28. Cuellar JM, Montesano PX and Carstens E: Role of TNF-alpha in sensitization of nociceptive dorsal horn neurons induced by application of nucleus pulposus to L5 dorsal root ganglion in rats. Pain 110: 578-587, 2004.

29. Murata Y, Nannmark U, Rydevik B, et al: The role of tumor necrosis factor-alpha in apoptosis of dorsal root ganglion cells induced by herniated nucleus pulposus in rats. Spine (Phila Pa 1976) 33: 155-162, 2008.

30. Bachmeier BE, Nerlich AG, Weiler C, et al: Analysis of tissue distribution of TNF-alpha, TNF-alpha-receptors, and the activating TNF-alpha-converting enzyme suggests activation of the TNF-alpha system in the aging intervertebral disc. Ann NY Acad Sci 1096: 44-54, 2007.

31. Brisby H, Olmarker K, Larsson K, et al: Proinflammatory cytokines in cerebrospinal fluid and serum in patients with disc herniation and sciatica. Eur Spine J 11: 62-66, 2002.

32. Pérez-Morga D, Vanhollebeke B, Paturiaux-Hanocq F, et al: Apolipoprotein L-I promotes trypanosome lysis by forming pores in lysosomal membranes. Science 309: 469-472, 2005.

33. Vanhamme L, Paturiaux-Hanocq F, Poelvoorde P, et al: Apolipoprotein L-I is the trypanosome lytic factor of human serum. Nature 422: 83-87, 2003.

34. Wan G, Zhaoriqetu S, Liu Z, et al: Apolipoprotein L1, a novel Bcl-2 homology domain 3-only lipid-binding protein, induces autophagic cell death. J Biol Chem 283: 21540-21549, 2008.

35. Clemmensen I, Petersen LC and Kluft C: Purification and characterization of a novel, oligomeric, plasminogen kringle 4 binding protein from human plasma: tetranectin. Eur J Biochem 156: 327-333, 1986.

36. Caterer NR, Graversen JH, Jacobsen C, et al: Specificity determinants in the interaction of apolipoprotein(a) kringles with tetranectin and LDL. Biol Chem 383: 1743-1750, 2002.

37. Kluft C, Jie AF, Los P, et al: Functional analogy between lipoprotein(a) and plasminogen in the binding to the kringle 4 binding protein, tetranectin. Biochem Biophys Res Commun 161: 427-433, 1989.

38. Mogues T, Etzerodt M, Hall C, et al: Tetranectin binds to the kringle 1-4 form of angiostatin and modifies its functional activity. J Biomed Biotechnol 2004: 73-78, 2004. 
39. Westergaard UB, Andersen MH, Heegaard CW, et al: Tetranectin binds hepatocyte growth factor and tissue-type plasminogen activator. Eur J Biochem 270: 1850-1854, 2003.

40. Borregaard N, Christensen L, Bejerrum OW, et al: Identification of a highly mobilizable subset of human neutrophil intracellular vesicles that contains tetranectin and latent alkaline phosphatase. J Clin Invest 85: 408-416, 1990.

41. Clemmensen I, Lund LR, Christensen L and Andreasen PA: A tetranectin-related protein is produced and deposited in extracellular matrix by human embryonal fibroblasts. Eur J Biochem 195: 735-741, 1991.

42. Nielsen H, Clemmensen I and Kharazmi A: Tetranectin: a novel secretory protein from human monocytes. Scand J Immunol 37: 39-42, 1993

43. Wewer UM, Iba K, Durkin ME, et al: Tetranectin is a novel marker for myogenesis during embryonic development, muscle regeneration, and muscle cell differentiation in vitro. Dev Biol 200: 247-259, 1998.

44. Wewer UM, Ibaraki K, Schjørring P, et al: A potential role for tetranectin in mineralization during osteogenesis. J Cell Biol 127 $1767-1775,1994$
45. Christensen L: The distribution of fibronectin, laminin and tetranectin in human breast cancer with special attention to the extracellular matrix. APMIS Suppl 26: 1-39, 1992.

46. Kamper EF, Kopeikina LT, Trontzas P, et al: Comparative study of tetranectin levels in serum and synovial fluid of patients with rheumatoid arthritis, seronegative spondylarthritis and osteoarthritis. Clin Rheumatol 17: 318-324, 1998.

47. Kluft C, Los P, Clemmensen I, et al: Quantitation of plasma levels of tetranectin - effects of oral contraceptives, pregnancy, treatment with L-asparaginase and liver cirrhosis. Thromb Haemost 62: 792-796, 1989.

48. Xu N and Dahlbäck B: A novel human apolipoprotein (apoM), J Biol Chem 274: 31286-31290, 1999.

49. Xu N, Hurtig M, Zhang XY, Ye Q and Nilsson-Ehle P: Transforming growth factor-beta down-regulates apolipoprotein M in HepG2 cells. Biochim Biophys Acta 1683: 33-37, 2004.

50. Luo G, Zhang X, Nilsson-Ehle P and Xu N: Apolipoprotein M. Lipids Health Dis 3: 21, 2004. 